\title{
A clinically isolated syndrome: butterfly glioma mimic
}

\author{
Ramshekhar Menon ${ }^{1}$, Bejoy Thomas ${ }^{2}$, Hariharan Venkat Easwer ${ }^{3}$, Samvedam Sandhyamani ${ }^{4}$, Amita Nair ${ }^{4}$, \\ Muralidharan Nair ${ }^{1}$ \\ ${ }^{1}$ Department of Neurology, Sree Chitra Tirunal Institute for Medical Sciences and Technology, Trivandrum 695011, Kerala, India. \\ ${ }^{2}$ Department of Imaging Sciences and Interventional Radiology, Sree Chitra Tirunal Institute for Medical Sciences and Technology, \\ Trivandrum 695011, Kerala, India. \\ ${ }^{3}$ Department of Neurosurgery, Sree Chitra Tirunal Institute for Medical Sciences and Technology, Trivandrum 695011, Kerala, India. \\ ${ }^{4}$ Department of Pathology, Sree Chitra Tirunal Institute for Medical Sciences and Technology, Trivandrum 695011, Kerala, India.
}

\section{A B S T R A C T}

The report explores a unique and treatable "butterfly"-glioma mimic and the neuroimaging characteristics that help to diagnose this entity. A 35-year-old patient presented with subacute-onset, progressive frontal lobe dysfunction followed by features of raised intracranial pressure. Neuroimaging features were consistent with a "butterfly" lesion that favored the possibility of a gliomatosis cerebri with significant edema and marked corpus callosum and fornix thickening. Contrast-enhanced and perfusion images revealed a confluent tumefactive lesion with a characteristic "broken-ring" pattern of enhancement, mass-effect and low perfusion; features favoring an alternative inflammatory pathology. This was peculiar as calloso-forniceal involvement of this nature has not been previously reported in inflammatory demyelinating mass lesions. This was confirmed as a tumefactive demyelination on histopathology. Following treatment, on clinical and imaging follow-up, significant resolution was evident suggesting a monophasic illness. This case highlights the stringent clinico-radiological-pathological approach required in the evaluation and management of butterfly lesions despite the striking imaging appearances. Tumefactive demyelination in this patient represents a clinically isolated syndromic presentation of an inflammatory pathology that can resemble gliomatosis cerebri. These "butterfly"-glioma mimics are scarcely reported in the literature, are eminently treatable with variable prognosis and prone for relapse.

Key words: Butterfly lesions, glioma, tumefactive demyelination

\section{INTRODUCTION}

Confluent "butterfly" lesions involving the corpus callosum often portend a poor prognosis in patients presenting with acute-subacute encephalopathy, with or without focal signs and raised intracranial pressure. Gliomatosis cerebri is often the primary pathological substrate considered in this situation. This report highlights an important mimic of "butterfly"-glioma.

\section{CASE REPORT}

A 35-year-old well-educated previously healthy male with no medical co-morbidities presented with complaints of behavioral disturbances for 1 month

\begin{tabular}{|l|l|}
\hline \multicolumn{2}{|c|}{ Access this article online } \\
\hline Quick Response Code: & \\
\hline & Website: \\
\hline & Www.nnjournal.net \\
\cline { 2 - 3 } & DOI: \\
\hline
\end{tabular}

prior to the index evaluation. The patient was observed by relatives to be apathetic, occasionally agitated and argumentative and over 15 days developed social dysinhibition, urinary and fecal incontinence. Excessive day time somnolence was also noted. The relatives also noted progressive inattention and memory impairment for a week prior to presentation. There was no history of any fever, headache, vomiting, loss of consciousness, seizures, hallucinations, delusions, focal limb weakness, imbalance nor any history suggestive of cranial nerve involvement.

Clinical examination revealed a conscious oriented patient with normal general physical examination findings. Mini Mental Status Examination score was 26/30 with impaired attention. There was a distinct lack of insight and impaired abstract thinking and judgment, with normal performance on tests of executive function. The rest of the cognitive evaluation was normal. There was no evidence of papilledema, ataxia or extrapyramidal signs. The plantar responses were bilaterally extensor consistent with a bipyramidal dysfunction. 
A clinical impression of a frontal space occupying lesion (SOL) was considered in view of a history suggestive of mesial and orbitofrontal dysfunction with suspicion of white-matter (WM) involvement in view of pyramidal signs. The initial magnetic resonance imaging (MRI) [Figure 1] revealed an ill-defined butterfly shaped intra axial SOL involving bilateral frontal lobes as well as anterior insula with predominant involvement of subcortical deep WM and extension into bilateral caudate nuclei, genu and the anterior $1 / 3$ of body of corpus callosum. Moderate mass-effect over bilateral frontal horns of lateral ventricles was observed. The lesion was hyperintense on T2-weighted and hypointense on T1-weighted images with mild diffusion restriction over the periphery. Peripheral enhancement was noted on intravenous gadolinium administration. There was no MR evidence of calcification or hemorrhage within the lesion. MR spectroscopy (MRS) from the intermediate part of the lesion revealed elevated choline peak, reduced $\mathrm{N}$-acetyl aspartate (NAA) and the presence of lactate; however perfusion values were noted to be low in the lesion.

On admission, the patient was noted to develop rapidly progressive encephalopathy with features of raised intracranial pressure in the form of bradycardia, hypersomnolence and hypertension. With the imaging consideration of an intermediate-high grade SOL and gliomatosis cerebri high on the cards in view of a butterfly-patterned lesion with calloso-forniceal thickening [Figure 1a], a neuronavigation-guided biopsy and as an alternative a frontal decompressive procedure was considered. The frozen-section specimen and histopathology report [Figure 2] was consistent with tumefactive demyelination hence decompression was not performed. Such a presentation mimicking a butterfly glioma is extremely rare. The patient was treated with pulse methyl predisolone followed by oral prednisolone that was administered in a dose of $1 \mathrm{mg} / \mathrm{kg}$ for 8 weeks followed by slow taper and cessation over 1 year. Prior to discharge evoked potentials, and cerebrospinal fluid studies including oligoclonal bands were negative thereby making multiple sclerosis (MS) less likely. Dramatic clinical improvement was noted, and the patient returned to the premorbid personality with normal neuropsychological performance 3 months into treatment. Serial MRI [Figure 3] verified gradual resolution of the WM hyperintensities and contrast enhancement with development of minimal bifrontal atrophy. MRS at 1 year showed reduction in the choline peak with reduced NAA and no evident lactate peak.

\section{DISCUSSION}

The neuroimaging characteristics distinctive in the pattern of tumefactive demyelination in the index case include the butterfly configuration, forniceal thickening and features of a "mass-effect" with a clinical presentation akin to a butterfly glioma; prominent differentiating features being the enhancement and perfusion patterns. The differential diagnosis of acute-subacute acquired "butterfly lesions" involving the corpus callosum as seen in the patient represents a challenge in itself with multimodal imaging playing a crucial role. A host of etiologies can be broadly grouped as: tumors such as glioma, lymphoma and metastasis; inflammatory demyelinating pathologies like tumefactive MS; infections such as progressive multifocal leukoencephalopathy and Whipple's disease; toxins leading to disseminated necrotizing leukoencephalopathy, e.g. intrathecal or systemic exposure to methotrexate or cytosine arabinoside and acute radiation necrosis. ${ }^{[1]}$ Imaging characteristics that potentially differentiate these conditions are depicted in Table 1. Clinico-radiological presentation of a non-neoplastic pathology like tumefactive demyelinating lesion (TDL) resembling a butterfly glioma is fraught with chance of a misdiagnosis (due to heterogeneity of imaging characteristics within the lesion itself).

Unlike in our patient, TDL tend to be circumscribed lesions with mild mass-effect or vasogenic edema. ${ }^{[2]}$ These typically involve the supra-tentorial WM although they may extend to involve the cortical gray matter with gyral edema. In a large series of 168 patients with biopsy confirmed central nervous system inflammatory demyelinating disease, frontal and parietal subcortical regions were most often affected and a butterfly configuration involving the corpus callosum was observed in only $12 \%$ of cases. ${ }^{[3]}$ Forniceal thickening was not described in this series and represents a unique observation in our patient as this deviated the impression towards a neoplastic etiology. Approximately half of TDL have pathological contrast enhancement, usually in the form of ring enhancement. ${ }^{[2,3]} \mathrm{A}$ variety of intracranial pathologies can present as a ring-enhancing lesion (REL) on MRI, including glioma, metastasis, lymphoma, radiation necrosis, infarct, abscess and tumefactive demyelination. Although less common in typical demyelination, REL are more likely to be biopsied in order to exclude these other pathologies that are mandatory from a treatment and prognostication point of view. In a recent series among the most prevalent pathologies associated with ring enhancement, demyelinating lesions of MS constituted a small number (6\%) and patterns of T2-weighted hypointensity are useful to differentiate between pathologies ${ }^{[4]}$ Figure $1 \mathrm{~b}$ and $\mathrm{c}$ demonstrate this pattern of enhancement in the patient. The enhancing portion of the ring is believed to represent the leading edge of demyelination and thus favors the WM side of 


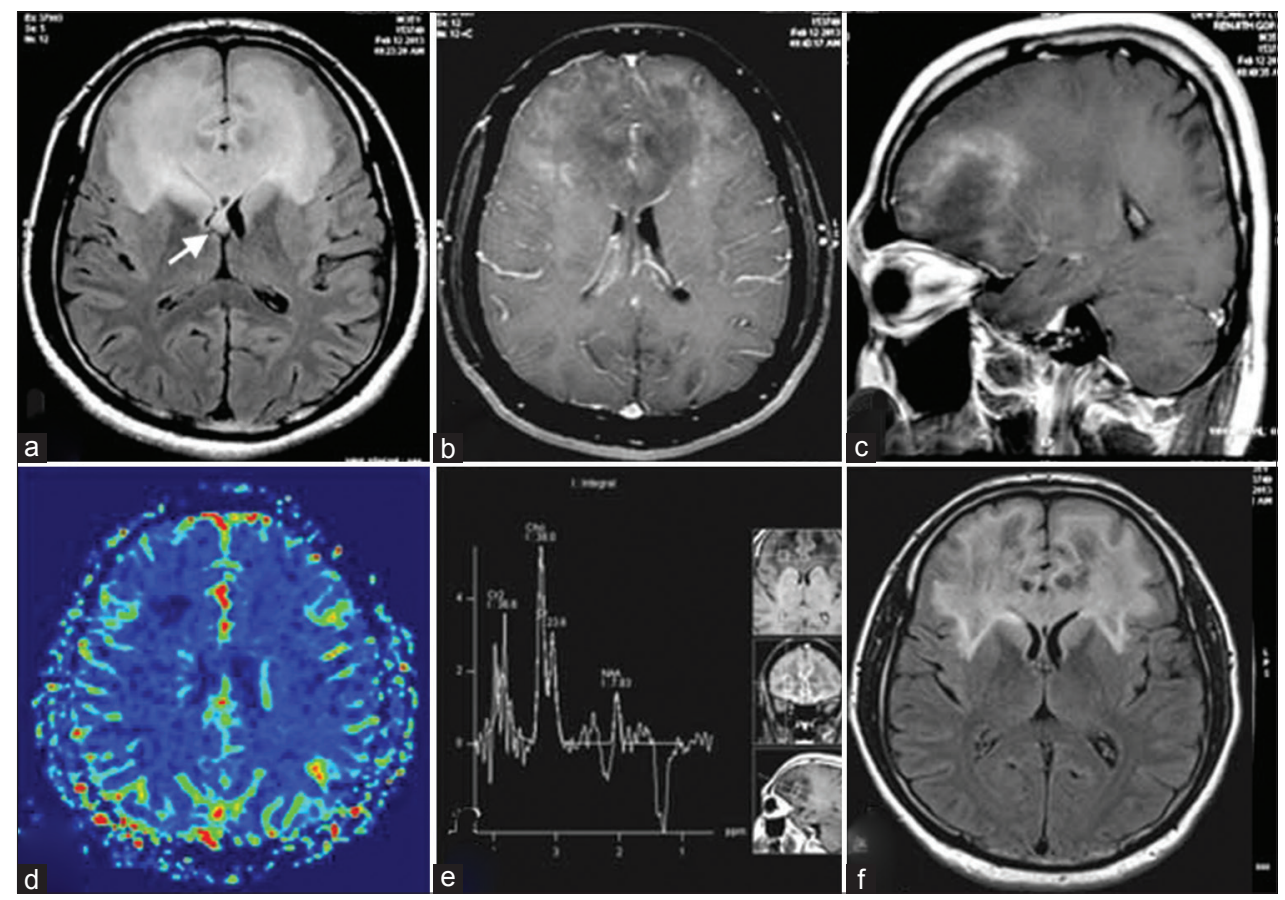

Figure 1: (a) Fluid-attenuated inversion recovery (FLAIR) axial image showing hyperintense lesion along the frontal subcortical white matter with callosal and forniceal involvement (arrow); ( $b$ and c) typical open rim pattern of enhancement on axial and saggital gadolinium T1-weighted images; (d) perfusion imaging indicating low perfusion; (e) magnetic resonance spectroscopy over intermediate zone that demonstrates choline peak with reduction in $\mathrm{N}$-acetyl aspartate; (f) immediate postbiopsy FLAIR image after pulse steroid therapy demonstrating reduction in edema and mass-effect
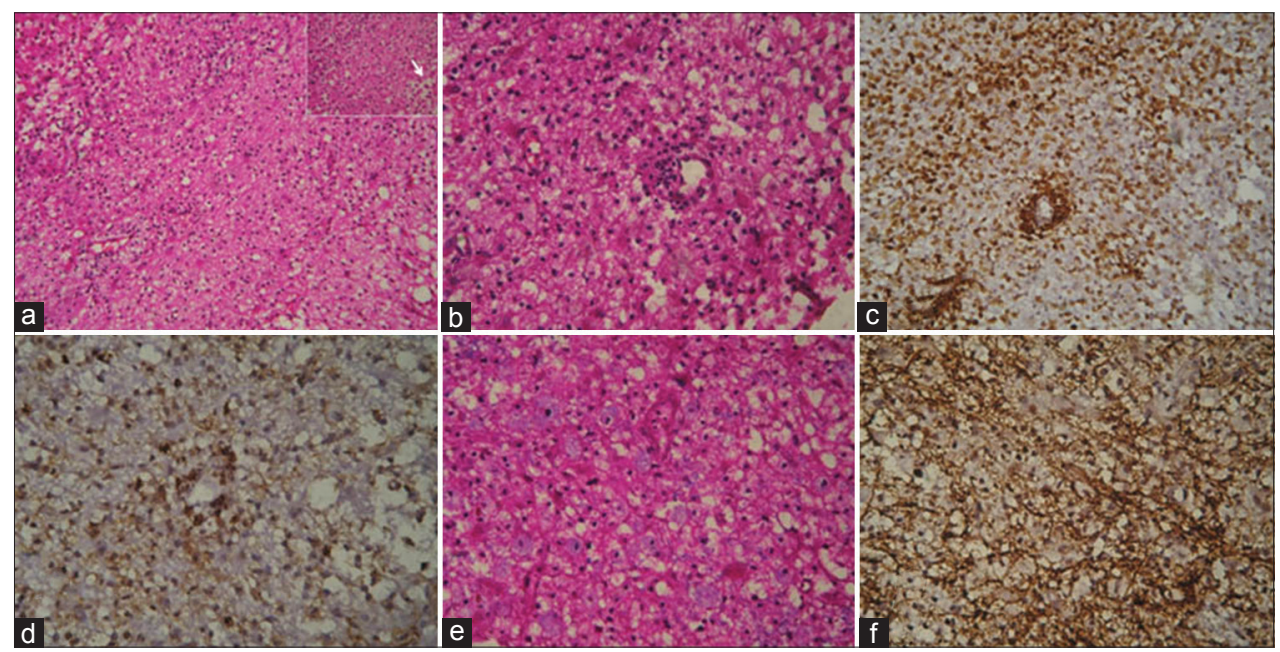

Figure 2: (a) Microscopy shows sheets of foamy macrophages with reactive astrocytes (HE, $\times 40)$; (inset arrow) sheets of foamy macrophages (HE, $\times 400)$; (b) perivascular and diffusely scattered lymphocytes (HE, x100); (c) CD68 showing perivascular and diffuse infiltrate of macrophages ( $\times 100)$; (d) CD3 showing perivascular and diffuse T cell infiltate (x100); (e) luxol fast blue hematoxylin stain shows macrophages with ingested myelin (blue stained, $\times 400)$; (f) preserved staining of neurofilament protein $(\times 100)$
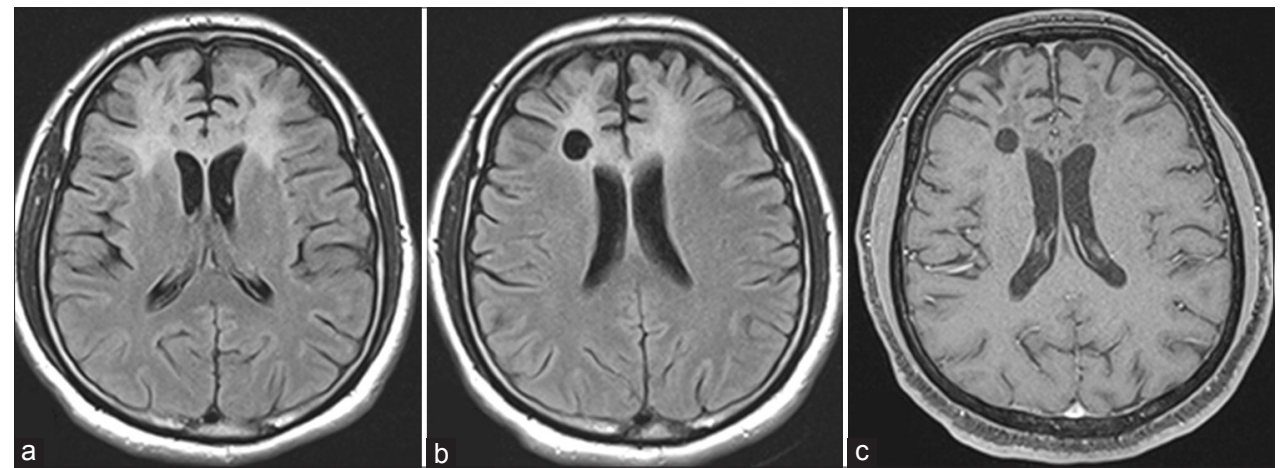

Figure 3: (a) Serial magnetic resonance imaging at 4th month of follow-up showing persistence of fluid-attenuated inversion recovery (FLAIR) hyperintensity with early overlying bifrontal atrophy; (b) FLAIR image at 1 year of follow-up demonstrating marked reduction in hyperintensity and significant atrophy; (c) T1-weighted gadolinium-enhanced image at 1 year showing no contrast enhancement in contrast to Figure $1 \mathrm{~b}$ and $\mathrm{c}$ 
Table I:Approach to differential diagnosis of "butterfly" lesions on MRI

\begin{tabular}{|c|c|c|c|c|c|}
\hline Pathology & TIWI & T2WI & Gadolinium contrast & $\begin{array}{l}\text { MR perfusion } \\
\text { ( } \mathrm{rCBV} \text { ) }\end{array}$ & Special features \\
\hline GBM & Iso-/ $\downarrow$ & $\begin{array}{l}\uparrow / \text { Iso-with solid } \\
\text { and cystic } \\
\text { components }\end{array}$ & $\begin{array}{l}\text { Heterogenous enhancement } \\
\text { of solid portion; REL }\end{array}$ & $\uparrow$ & $\begin{array}{l}\text { MRS: choline, lipid, lactate peaks } \\
\text { DWI/ADC: ++ in solid portion } \\
\text { GRE: hemorrhagic }\end{array}$ \\
\hline Lymphoma & Iso-/ $\downarrow$ & Iso-/ $\downarrow$ & $\begin{array}{l}\text { Homogenous; REL in } \\
\text { immunocompromised }\end{array}$ & $N / \downarrow$ & $\begin{array}{l}\text { MRS: same as GBM } \\
\text { DWI/ADC: }++\end{array}$ \\
\hline Metastasis & $\begin{array}{l}\text { Iso-/ } \\
\text { hemorrhagic/ } \downarrow\end{array}$ & $\uparrow$ & $\begin{array}{l}\text { Variable enhancement } \\
\text { patterns (solid, REL, irregular, } \\
\text { homogenous, mixed) }\end{array}$ & $N / \uparrow$ & $\begin{array}{l}\text { MRS: choline peak } \\
\text { DWI/ADC: variable } \\
\text { GRE: variable } \\
\text { Lesion location at GWM junctions }\end{array}$ \\
\hline PML & $\downarrow$ & $\uparrow$ & $\begin{array}{l}\text { Mildperipheral enhancement; } \\
\uparrow \text { during IRIS }\end{array}$ & $\downarrow$ & $\begin{array}{l}\text { MRS: NAA } \downarrow \text { in WM with } U \text { fiber } \\
\text { scalloping }\end{array}$ \\
\hline $\begin{array}{l}\text { Diffuse } \\
\text { necrotizing leuko- } \\
\text { encephalopathy }\end{array}$ & $\downarrow$ & $\uparrow$ & Rare peripheral enhancement & $N / \downarrow$ & $\begin{array}{l}\text { DWI/ADC:++ } \\
\text { Usually evanescent, diffuse/ } \\
\text { multi-focal; peri-ventricular with } \\
\text { sparing of } U \text { fibers }\end{array}$ \\
\hline $\begin{array}{l}\text { Acute radiation } \\
\text { necrosis }\end{array}$ & Iso-/ $\downarrow$ & $\begin{array}{l}\text { Central- } \uparrow \\
\text { Solid part- } \downarrow\end{array}$ & REL around necrosis & $N / \uparrow$ & $\begin{array}{l}\text { DWI/ADC:++ } \\
\text { GRE: micro-hemorrhages and } \\
\text { calcification }\end{array}$ \\
\hline $\begin{array}{l}\text { Whipple's } \\
\text { disease }\end{array}$ & $\downarrow$ & $\uparrow$ & $\begin{array}{l}\text { Punctate, incomplete } \\
\text { enhancement }\end{array}$ & $N / \downarrow$ & $\begin{array}{l}\text { Location: thalami, WM and } \\
\text { brainstem }\end{array}$ \\
\hline $\begin{array}{l}\text { Inflammatory } \\
\text { demyelination } \\
\text { (tumefactive) }\end{array}$ & $\downarrow$ & $\uparrow$ & "Broken-ring" or closed ring & $\downarrow$ & $\begin{array}{l}\text { Variable MRS and DWI/ADC } \\
\text { values within lesion }\end{array}$ \\
\hline
\end{tabular}

T1WI: T1-weighted imaging; T2WI: T2-weighted imaging; rCBV: regional cerebral blood volume; REL: ring-enhancing lesion; DWI: diffusion weighted imaging;

ADC: apparent diffusion coefficient; $\uparrow$ : hyperintensity; $\downarrow$ : hypointensity; ++: diffusion restriction; N: normal; MRS: magnetic resonance spectroscopy; GWM: grey-white matter; WM: white matter; GRE: gradient echo imaging; IRIS: immune reconstitution inflammatory syndrome; MRI: magnetic resonance imaging; GBM: glioblastoma multiforme; NAA: N-acetyl aspartate; PML: progressive multifocal leukoencephalopathy

the lesion. ${ }^{[2]}$ The central non-enhancing core represents a more chronic phase of the inflammatory process. Reduced perfusion may be another suggestive feature of $\mathrm{TDL},{ }^{[5]}$ however exclusion of a moderate grade butterfly glioma was mandatory in the patient. Demyelination co-existing with primary neoplasms has been reported rarely in the literature but the histopathology and subsequent clinico-radiological follow-up excluded this association ${ }^{[6]}$ In comparison with tumors and abscesses, edema in TDL is said to be proportionally minor relative to plaque size contrary to what was seen in our patient. ${ }^{[7]}$

Another case series emphasized the unique multimodal imaging characteristics in tumefactive demyelination. ${ }^{[8]}$ Two or three concentric distinct zones were noted on imaging with distinct metabolic and structural signature in most cases. Increase in the glutamine/ glutamate ratio and lactate was noted in tumefactive lesions. On TE $135 \mathrm{~ms}$, the central part showed variable Choline (Cho) and significantly low NAA. The intermediate area showed higher Cho and lower NAA compared to contralateral normal side as was seen in the patient. The outermost layer, which corresponded to the contrast enhancing areas on MRI, showed high Cho, lower NAA, and restricted diffusion. Follow-up imaging, as was seen in this series, showed a reduction in the extent of hyperintensities, however MRS showed persistent abnormalities.

While remission was seen 1 year into follow-up in our patient, it is important to note that only $17 \%$ of cases in Lucchinetti's series remained unifocal during radiological follow-up. ${ }^{[3]}$ Longitudinal follow-up had revealed eight developed definite MS, and one had isolated demyelinating syndrome by the last follow-up. The unifocal subgroup was more likely to have mass-effect and edema associated with the biopsied lesion on prebiopsy scan, compared with those who developed multifocal lesions. One cannot reliably exclude the fact that with a large multilobar lesion as seen in our case, the area selected for biopsy is more likely due to surgical bias and may not be representative of the true pathology. The serial follow-up makes alternative possibilities remote. Patient age, clinical course prior to biopsy or disability status at last follow-up have not been found to differ between patients with or without a butterfly lesion. ${ }^{[3]}$

In a review of 31 cases, Kepes ${ }^{[9]}$ proposed that TDLs represent an intermediate lesion between those typically seen with MS and acute disseminated encephalomyelitis. Pathologically, these lesions are indistinguishable from typical MS plaques and are characterized by infiltrating foamy macrophages intermingled between reactive astrocytes [Figure 2]. ${ }^{[10]}$ Significant quantities of lipid may accumulate within the plaques as a result of myelin breakdown. The pathologic diagnosis may be challenging based on the initial frozen-section specimen when the primary suspicion is malignancy. In our patient, absence of features such as hyperchromatic nuclear morphology, uneven pattern of distribution of astrocytes, atypical 
mitotic figures, necrosis and endothelial proliferation, correlated to the subsequent clinico-radiological profile, glioma co-existing with TDL could be reliably excluded. ${ }^{[11]}$ Our case highlights the importance of meticulous radiopathological inputs required into analysis of butterfly multifocal lesions that is the key to guide subsequent management of an evidently treatable condition.

\section{REFERENCES}

1. Bourekas EC, Varakis K, Bruns D, Christoforidis GA, Baujan M, Slone HW, Kehagias D. Lesions of the corpus callosum: MR imaging and differential considerations in adults and children. AJR Am J Roentgenol 2002;179:251-7.

2. Kobayashi M, Shimizu Y, Shibata N, Uchiyama S. Gadolinium enhancement patterns of tumefactive demyelinating lesions: correlations with brain biopsy findings and pathophysiology. J Neurol 2014;261:1902-10.

3. Lucchinetti CF, Gavrilova RH, Metz I, Parisi JE, Scheithauer BW, Weigand S, Thomsen K, Mandrekar J, Altintas A, Erickson BJ, Konig F, Giannini C, Lassmann H, Linbo L, Pittock SJ, Bruck W. Clinical and radiographic spectrum of pathologically confirmed tumefactive multiple sclerosis. Brain 2008;131:1759-75.

4. Schwartz KM, Erickson BJ, Lucchinetti C. Pattern of T2 hypointensity associated with ring-enhancing brain lesions can help to differentiate pathology. Neuroradiology 2006;48:143-9.

5. Jain R, Ellika S, Lehman NL, Scarpace L, Schultz LR, Rock JP,
Rosenblum M, Mikkelsen T. Can permeability measurements add to blood volume measurements in differentiating tumefactive demyelinating lesions from high grade gliomas using perfusion $\mathrm{CT}$ ? J Neurooncol 2010;97:383-8.

6. Roemer SF, Scheithauer BW, Varnavas GG, Lucchinetti CF Tumefactive demyelination and glioblastoma: a rare collision lesion. Clin Neuropathol 2011;30:186-91.

7. Altintas A, Petek B, Isik N, Terzi M, Bolukbasi F, Tavsanli M, Saip S Boz C, Aydin T, Arici-Duz O, Ozer F, Siva A. Clinical and radiological characteristics of tumefactive demyelinating lesions: follow-up study. Mult Scler 2012;18:1448-53.

8. Saini J, Chatterjee S, Thomas B, Kesavadas C. Conventional and advanced magnetic resonance imaging in tumefactive demyelination. Acta Radiol 2011;52:1159-68.

9. Kepes JJ. Large focal tumor-like demyelinating lesions of the brain: intermediate entity between multiple sclerosis and acute disseminated encephalomyelitis? A study of 31 patients. Ann Neurol 1993;33:18-27.

10. Neelima R, Krishnakumar K, Nair MD, Kesavadas C, Hingwala DR Radhakrishnan VV, Nair SS. Tumefactive demyelinating lesions: a clinicopathological correlative study. Indian J Pathol Microbiol 2012;55:496-500.

11. Donev K, Scheithauer BW. Pseudoneoplasms of the nervous system. Arch Pathol Lab Med 2010;134:404-16.

Cite this article as: Menon R, Thomas B, Easwer HV, Sandhyamani S, Nair A Nair M. A clinically isolated syndrome: butterfly glioma mimic. Neuroimmunol Neuroinflammation 2015;2(3):174-8

Source of Support: Nil. Conflict of Interest: No.

Received: 30-10-2014; Accepted: 11-02-2015 